\title{
Two Efficient Generalized Laguerre Spectral Algorithms for Fractional Initial Value Problems
}

\author{
D. Baleanu, ${ }^{1,2,3}$ A. H. Bhrawy, ${ }^{4,5}$ and T. M. Taha ${ }^{5}$ \\ ${ }^{1}$ Department of Mathematics and Computer Sciences, Faculty of Arts and Sciences, Cankaya University, \\ Eskisehir Yolu 29.Km, 06810 Ankara, Turkey \\ ${ }^{2}$ Department of Chemical and Materials Engineering, Faculty of Engineering, King Abdulaziz University, Jeddah, Saudi Arabia \\ ${ }^{3}$ Institute of Space Sciences, Magurele-Bucharest, Romania \\ ${ }^{4}$ Department of Mathematics, Faculty of Science, King Abdulaziz University, Jeddah, Saudi Arabia \\ ${ }^{5}$ Department of Mathematics, Faculty of Science, Beni-Suef University, Beni Suef, Egypt
}

Correspondence should be addressed to A. H. Bhrawy; alibhrawy@yahoo.co.uk

Received 15 April 2013; Accepted 21 May 2013

Academic Editor: Soheil Salahshour

Copyright (C) 2013 D. Baleanu et al. This is an open access article distributed under the Creative Commons Attribution License, which permits unrestricted use, distribution, and reproduction in any medium, provided the original work is properly cited.

We present a direct solution technique for approximating linear multiterm fractional differential equations (FDEs) on semiinfinite interval, using generalized Laguerre polynomials. We derive the operational matrix of Caputo fractional derivative of the generalized Laguerre polynomials which is applied together with generalized Laguerre tau approximation for implementing a spectral solution of linear multiterm FDEs on semi-infinite interval subject to initial conditions. The generalized Laguerre pseudospectral approximation based on the generalized Laguerre operational matrix is investigated to reduce the nonlinear multiterm FDEs and its initial conditions to nonlinear algebraic system, thus greatly simplifying the problem. Through several numerical examples, we confirm the accuracy and performance of the proposed spectral algorithms. Indeed, the methods yield accurate results, and the exact solutions are achieved for some tested problems.

\section{Introduction}

Fractional calculus has been used to develop accurate models of many phenomena of science, engineering, economics, and applied mathematics. These models are found to be best described by FDEs [1-4].

One of the best methods, in terms of the accuracy, for investigating the numerical solution of various kinds of differential equations is spectral method (see, for instance, [5-8]). Because all types of spectral methods are global and numerical computational methods, they are very convenient for approximating linear and nonlinear FDEs $[6,7,9]$. We refer also to recent numerical and analytical methods for solving FEEs [10-16].

In the last few years, theory and numerical solution of FDEs by using spectral methods have received an increasing attention. In this direction, Doha et al. [17] proposed an effective way to approximate solutions of linear and nonlinear multiterm FDEs with constant and variable coefficients using
Jacobi spectral approximation, in which they generalized the Chebyshev spectral methods [6] and quadrature Legendre tau method [9]; moreover, other very important cases can be obtained for that approach. Maleki et al. [18] proposed an efficient and accurate spectral collocation method based on shifted Legendre-Gauss quadrature nodes for solving fractional boundary value problems in finite interval. The authors of [19] used the spline functions methods for tackling the linear and nonlinear FDEs. Recently, Bhrawy et al. [20] investigated the fractional integrals of modified generalized Laguerre operational matrix to implement a numerical solution of the integrated form of the linear FDEs on semiinfinite interval. Furthermore, Yuzbasi [21] proposed a new collocation method based on Bessel functions to introduce an approximate solution of a class of FDEs. We refer also to the recent papers [22-26] where operational matrices of several orthogonal polynomials are developed for solving linear and nonlinear ODEs and FDEs. 
In this paper, the Caputo fractional derivative of generalized Laguerre operational matrix (GLOM) is stated and proved. The main aim of this paper is to extend the application of generalized Laguerre spectral tau method based on GLOM to develop a direct solution technique for the numerical solution of linear multi-term FDEs on a semi-infinite interval. Moreover, we develop the generalized Laguerre pseudo-spectral approximation based on the GLOM for reducing the nonlinear multi-term FDEs subject to nonhomogeneous initial conditions to a system of nonlinear algebraic equations. Finally, the accuracy of the proposed algorithms is demonstrated by test problems. The numerical results are given to show that the proposed spectral algorithms based on generalized Laguerre operational matrix of Caputo fractional derivatives are very effective for linear and nonlinear FDEs.

The outline of the paper is as follows. In Section 2, we present some preliminaries. Section is devoted to drive the GLOM of Caputo fractional derivative. In Section 4, we extend the generalized Laguerre spectral tau and pseudospectral approximations based on the GLOM of fractional derivative for solving multiorder linear and nonlinear FDEs. Some numerical experiments are presented in Section 5. Finally, we conclude the paper with some remarks.

\section{Some Basic Preliminaries}

The two most commonly used definitions are the RiemannLiouville operator and the Caputo operator. We give some definitions and properties of fractional derivatives and generalized Laguerre polynomials.

Definition 1. The fractional integral operator of RiemannLiouville sense is defined as

$$
\begin{gathered}
J^{\nu} f(x)=\frac{1}{\Gamma(\nu)} \int_{0}^{x}(x-t)^{\nu-1} f(t) d t, \quad \nu>0, x>0, \\
J^{0} f(x)=f(x) .
\end{gathered}
$$

Definition 2. The Caputo fractional derivatives is given by

$$
\begin{aligned}
& D^{v} f(x) \\
& =J^{m-v} D^{m} f(x) \\
& =\frac{1}{\Gamma(m-v)} \int_{0}^{x}(x-t)^{m-v-1} \frac{d^{m}}{d t^{m}} f(t) d t, \\
& \quad m-1<v<m, x>0,
\end{aligned}
$$

where $D^{m}$ is $m$ th order differential operator.

The Caputo fractional derivative operator satisfies

$$
D^{\nu} C=0, \quad(C \text { is a constant }),
$$

$$
\begin{aligned}
& D^{\nu} x^{\beta} \\
& =\left\{\begin{array}{ll}
0, & \text { for } \beta \in N_{0}, \beta<\lceil\nu\rceil, \\
\frac{\Gamma(\beta+1)}{\Gamma(\beta+1-\nu)} x^{\beta-\nu}, & \text { for } \beta \in N_{0}, \beta \geq\lceil\nu\rceil \text { or }
\end{array} \text {, } \beta \notin N>\lfloor\nu\rfloor,\right.
\end{aligned}
$$

where $\lceil\nu\rceil$ and $\lfloor\nu\rfloor$ are the ceiling and floor functions, respectively, while $N=\{1,2, \ldots\}$ and $N_{0}=\{0,1,2, \ldots\}$.

The Caputo's derivative operator is a linear operation:

$$
D^{\nu}(\lambda f(x)+\mu g(x))=\lambda D^{\nu} f(x)+\mu D^{v} g(x),
$$

where $\lambda$ and $\mu$ are constants.

We recall below some relevant properties of the generalized Laguerre polynomials (Szego [27] and Funaro [28]). let $\Lambda=(0, \infty)$ and let $w^{(\alpha)}(x)=x^{\alpha} e^{-x}$ be a weight function on $\Lambda$ in the usual sense. Define

$$
\begin{aligned}
& L_{w^{(\alpha)}}^{2}(\Lambda) \\
& \quad=\left\{v \mid v \text { is measurable on } \Lambda \text { and }\|v\|_{w^{(\alpha)}}<\infty\right\},
\end{aligned}
$$

with the inner product and norm

$$
\begin{gathered}
(u, v)_{w^{(\alpha)}}=\int_{\Lambda} u(x) v(x) w^{(\alpha)}(x) d x, \\
\|v\|_{w^{(\alpha)}}=(v, v)_{w^{(\alpha)}}^{1 / 2} .
\end{gathered}
$$

For $\alpha>-1$, the generalized Laguerre polynomials are given by

$$
L_{i}^{(\alpha)}(x)=\frac{1}{i !} x^{-\alpha} e^{x} \partial_{x}^{i}\left(x^{i+\alpha} e^{-x}\right), \quad i=1,2, \ldots
$$

According to [29] for $\alpha>-1$, we get

$$
\begin{aligned}
& \partial_{x} L_{i}^{(\alpha)}(x)=-L_{i-1}^{(\alpha+1)}(x), \\
& L_{i+1}^{(\alpha)}(x) \\
& =\frac{1}{i+1}\left[(2 i+\alpha+1-x) L_{i}^{(\alpha)}(x)-(i+\alpha) L_{i-1}^{(\alpha)}(x)\right], \\
& i=1,2, \ldots,
\end{aligned}
$$

where $L_{0}^{(\alpha)}(x)=1$ and $L_{1}^{(\alpha)}(x)=1+\alpha-x$.

The generalized Laguerre polynomials are the $L_{w^{(\alpha)}}^{2}(\Lambda)$ orthogonal system;

$$
\int_{0}^{\infty} L_{j}^{(\alpha)}(x) L_{k}^{(\alpha)}(x) w^{(\alpha)}(x) d x=h_{k} \delta_{j k}
$$

where $h_{k}=\Gamma(k+\alpha+1) / k$ ! from

The generalized Laguerre polynomials on $\Lambda$ are obtained

$$
L_{i}^{(\alpha)}(x)=\sum_{k=0}^{i}(-1)^{k} \frac{\Gamma(i+\alpha+1)}{\Gamma(k+\alpha+1)(i-k) ! k !} x^{k}, \quad i=0,1, \ldots
$$

The special value

$$
D^{q} L_{i}^{(\alpha)}(0)=(-1)^{q} \sum_{j=0}^{i-q} \frac{(i-j-1) !}{(q-1) !(i-j-q) !} L_{j}^{(\alpha)}(0), \quad i \geqslant q,
$$


where $L_{j}^{(\alpha)}(0)=\Gamma(j+\alpha+1) / \Gamma(\alpha+1) j$ !, will be of important use later, for treating the initial conditions of the given FDEs.

Let $u(x) \in L_{w^{(\alpha)}}^{2}(\Lambda)$, then $u(x)$ may be expressed in terms of generalized Laguerre polynomials as

$$
\begin{gathered}
u(x)=\sum_{j=0}^{\infty} a_{j} L_{j}^{(\alpha)}(x) \\
a_{j}=\frac{1}{h_{k}} \int_{0}^{\infty} u(x) L_{j}^{(\alpha)}(x) w^{(\alpha)}(x) d x, \quad j=0,1,2, \ldots
\end{gathered}
$$

In particular applications, the generalized Laguerre polynomials up to degree $N+1$ are considered. Then we have

$$
u_{N}(x)=\sum_{j=0}^{N} a_{j} L_{j}^{(\alpha)}(x) .
$$

We will present the Laguerre-Gauss quadrature. Let $\left\{x_{j}^{(\alpha)}, \omega_{j}^{(\alpha)}\right\}$ be the set of generalized Laguerre-Gauss quadrature nodes and weights:

$$
\int_{\Lambda} \phi(x) w^{(\alpha)}(x) d x=\sum_{j=0}^{N} \phi\left(x_{j}^{(\alpha)}\right) \omega_{j}^{(\alpha)} .
$$

For the generalized Laguerre-Gauss quadrature, $\left\{x_{j}^{(\alpha)}\right\}$ are the zeros of $L_{i+1}^{(\alpha)}(x)$, and

$$
\begin{aligned}
\omega_{j}^{(\alpha)} & =-\frac{\Gamma(i+\alpha+1)}{(i+1) ! L_{i}^{(\alpha)}\left(x_{j}^{(\alpha)}\right) \partial_{x} L_{i+1}^{(\alpha)}\left(x_{j}^{(\alpha)}\right)} \\
& =\frac{\Gamma(i+\alpha+1) x_{j}^{(\alpha)}}{(i+\alpha+1)(i+1) !\left[L_{i}^{(\alpha)}\left(x_{j}^{(\alpha)}\right)\right]^{2}}, \quad 0 \leq j \leq i .
\end{aligned}
$$

\section{GLOM of Fractional Derivatives}

Let $u(x) \in L_{w^{(\alpha)}}^{2}(\Lambda)$, and then $u(x)$ may be expanded using generalized Laguerre polynomials as

$$
\begin{gathered}
u(x)=\sum_{j=0}^{\infty} a_{j} L_{j}^{(\alpha)}(x), \\
a_{j}=\frac{1}{h_{k}} \int_{0}^{\infty} u(x) L_{j}^{(\alpha)}(x) w^{(\alpha)}(x) d x, \quad j=0,1,2, \ldots
\end{gathered}
$$

In particular applications, the generalized Laguerre polynomials up to degree $N+1$ are considered. Then we have

$$
u_{N}(x)=\sum_{j=0}^{N} a_{j} L_{j}^{(\alpha)}(x)=C^{T} \phi(x),
$$

where the vector $C$ and vector $\phi(x)$ are given by

$$
\begin{gathered}
C^{T}=\left[c_{0}, c_{1}, \ldots, c_{N}\right], \\
\phi(x)=\left[L_{0}^{(\alpha)}(x), L_{1}^{(\alpha)}(x), \ldots, L_{N}^{(\alpha)}(x)\right]^{T} .
\end{gathered}
$$

Then the derivative of the vector $\phi(x)$ can be expressed by

$$
\frac{d \phi(x)}{d x}=\mathbf{D}^{(1)} \phi(x)
$$

where $\mathbf{D}^{(1)}$ is the $(N+1) \times(N+1)$ operational matrix of the derivative given by

$$
\mathbf{D}^{(1)}=-\left(\begin{array}{cccccccc}
0 & 0 & 0 & 0 & 0 & \cdots & 0 & 0 \\
1 & 0 & 0 & 0 & 0 & \cdots & 0 & 0 \\
1 & 1 & 0 & 0 & 0 & \cdots & 0 & 0 \\
1 & 1 & 1 & 0 & 0 & \cdots & 0 & 0 \\
1 & 1 & 1 & 1 & 0 & \cdots & 0 & 0 \\
\vdots & \vdots & \vdots & \vdots & \vdots & \cdots & \vdots & \vdots \\
1 & 1 & 1 & 1 & 1 & \cdots & 1 & 0
\end{array}\right) .
$$

By using (21), it is clear that

$$
\frac{d^{n} \phi(x)}{d x^{n}}=\left(\mathbf{D}^{(1)}\right)^{n} \phi(x)
$$

where $n \in N$ and the superscript in $\mathbf{D}^{(1)}$ denotes matrix powers. Thus

$$
\mathbf{D}^{(n)}=\left(\mathbf{D}^{(1)}\right)^{n}, \quad n=1,2, \ldots
$$

Lemma 3. Let $L_{i}^{(\alpha)}(x)$ be a generalized Laguerre polynomial Then

$$
D^{\nu} L_{i}^{(\alpha)}(x)=0, \quad i=0,1, \ldots,\lceil\nu\rceil-1, \nu>0
$$

In the following theorem we prove the operational matrix of Caputo fractional derivative for the generalized Laguerre vector (20).

Theorem 4. Suppose $v>0$, the fractional derivative of order $\nu$ of $\phi(x)$ is given by

$$
D^{\nu} \phi(x) \simeq \mathbf{D}^{(\nu)} \phi(x),
$$

where $\mathbf{D}^{(v)}$ is the $(N+1) \times(N+1)$ operational matrix of Caputo fractional derivative and is given by

$$
\begin{gathered}
\mathbf{D}^{(\nu)} \\
=\left(\begin{array}{ccccc}
0 & 0 & 0 & \cdots & 0 \\
\vdots & \vdots & \vdots & \cdots & \vdots \\
0 & 0 & 0 & \cdots & 0 \\
S_{\nu}(\lceil\nu\rceil, 0) & S_{\nu}(\lceil\nu\rceil, 1) & S_{\nu}(\lceil\nu\rceil, 2) & \cdots & S_{\nu}(\lceil\nu\rceil, N) \\
\vdots & \vdots & \vdots & \cdots & \vdots \\
S_{\nu}(i, 0) & S_{\nu}(i, 1) & S_{\nu}(i, 2) & \cdots & S_{\nu}(i, N) \\
\vdots & \vdots & \vdots & \cdots & \vdots \\
S_{\nu}(N, 0) & S_{\nu}(N, 1) & S_{\nu}(N, 2) & \cdots & S_{\nu}(N, N)
\end{array}\right),
\end{gathered}
$$


where

$$
\begin{aligned}
& S_{\nu}(i, j) \\
& =\sum_{k=\lceil\nu\rceil \ell=0}^{i} \sum^{j}\left((-1)^{k+\ell} j ! \Gamma(i+\alpha+1)\right. \\
& \quad \times \Gamma(k-v+\alpha+\ell+1)) \\
& \quad \times((i-k) !(j-\ell) ! \ell ! \Gamma(k-v+1) \\
& \quad \times \Gamma(k+\alpha+1) \Gamma(\alpha+\ell+1))^{-1} .
\end{aligned}
$$

Proof. Applying (4) to (11) gives

$$
\begin{aligned}
& D^{\nu} L_{i}^{(\alpha)}(x) \\
&= \sum_{k=0}^{i}(-1)^{k} \frac{\Gamma(i+\alpha+1)}{(i-k) ! k ! \Gamma(k+\alpha+1)} D^{\nu} x^{k} \\
&= \sum_{k=\lceil\nu\rceil}^{i}(-1)^{k} \frac{\Gamma(i+\alpha+1)}{(i-k) ! \Gamma(k-\nu+1) \Gamma(k+\alpha+1)} x^{k-\nu}, \\
& i=\lceil\nu\rceil, \ldots, N .
\end{aligned}
$$

Now, $x^{k-\gamma}$ can be approximated by $N+1$ terms of the generalized Laguerre polynomials to get

$$
x^{k-v}=\sum_{j=0}^{N} b_{k j} L_{j}^{(\alpha)}(x),
$$

where $b_{j}$ is directly obtained from (18), and

$$
b_{j}=\sum_{\ell=0}^{j}(-1)^{\ell} \frac{j ! \Gamma(k-v+\alpha+\ell+1)}{(j-\ell) !(\ell) ! \Gamma(\ell+\alpha+1)} .
$$

Employing (29)-(31) yields

$$
D^{\nu} L_{i}^{(\alpha)}(x)=\sum_{j=0}^{N} S_{\nu}(i, j) L_{j}^{(\alpha)}(x), \quad i=\lceil\nu\rceil, \ldots, N,
$$

where

$$
\begin{aligned}
S_{\nu}(i, j)=\sum_{k=\lceil\nu\rceil \ell=0}^{i} \sum_{\ell=1}^{j}( & -1)^{k+\ell} j ! \Gamma(i+\alpha+1) \\
& \times \Gamma(k-v+\alpha+\ell+1)) \\
( & (i-k) !(j-\ell) ! \ell ! \\
& \times \Gamma(k-v+1) \Gamma(k+\alpha+1) \\
& \times \Gamma(\alpha+\ell+1))^{-1} .
\end{aligned}
$$

Accordingly, (32) can be written in a vector form as follows:

$$
\begin{array}{r}
D^{\nu} L_{i}^{(\alpha)}(x) \\
\simeq\left[S_{\nu}(i, 0), S_{\nu}(i, 1), S_{\nu}(i, 2), \ldots, S_{\nu}(i, N)\right] \phi(x), \\
i=\lceil\nu\rceil, \ldots, N .
\end{array}
$$

Also according to Lemma 3, we can write

$$
D^{\nu} L_{i}^{(\alpha)}(x) \simeq[0,0,0, \ldots, 0] \phi(x), \quad i=0,1, \ldots,\lceil\nu\rceil-1 .
$$

A combination of (34) and (35) leads to the desired result.

Remark 5. In the case of $v=n \in N$, Theorem 4 gives the same result as (23).

\section{Applications of GLOM for Multiterm FDEs}

In this section, we are interested in using GLOM in combination with two types of spectral methods for solving linear and nonlinear FDEs.

4.1. Linear Multiorder FDEs. Here, we propose a direct solution technique to approximate linear multi-term FDEs with constant coefficients using the generalized Laguerre tau method in combination with GLOM.

Consider the linear multi-order FDE

$$
D^{v} u(x)=\sum_{j=1}^{k} \gamma_{j} D^{\beta_{j}} u(x)+\gamma_{k+1} u(x)+g(x), \quad \text { in } \Lambda,
$$

with initial conditions

$$
u^{(i)}(0)=d_{i}, \quad i=0, \ldots, m-1,
$$

where $\gamma_{j}(j=1, \ldots, k+1)$ are constants and $m-1<$ $v \leq m, 0<\beta_{1}<\beta_{2}<\cdots<\beta_{k}<v$. Also $D^{v} u(x) \equiv$ $u^{(v)}(x)$ is $v$ order of Caputo fractional derivative for $u(x)$, $d_{i}(i=0, \ldots, m-1)$ are the initial values, and $g(x)$ is a source function.

To solve the fractional FDE (36)-(37), we approximate $u(x)$ and $g(x)$ by generalized Laguerre polynomials as

$$
\begin{aligned}
& u(x) \simeq \sum_{i=0}^{N} c_{i} L_{i}^{(\alpha)}(x)=C^{T} \phi(x), \\
& g(x) \simeq \sum_{i=0}^{N} g_{i} L_{i}^{(\alpha)}(x)=G^{T} \phi(x),
\end{aligned}
$$

where vector $G=\left[g_{0}, \ldots, g_{N}\right]^{T}$ is known. have

By using Theorem 4 (relation equations (26), and (38)) we

$$
\begin{gathered}
D^{\nu} u(x) \simeq C^{T} D^{\nu} \phi(x)=C^{T} D^{(\nu)} \phi(x), \\
D^{\beta_{j}} u(x) \simeq C^{T} D^{\beta_{j}} \phi(x)=C^{T} D^{\left(\beta_{j}\right)} \phi(x), \quad j=1, \ldots, k .
\end{gathered}
$$

Making use of (38)-(40), the residual $R_{N}(x)$ for (36) can be given from

$$
R_{N}(x)=\left(C^{T} D^{(\nu)}-C^{T} \sum_{j=1}^{k} \gamma_{j} \mathbf{D}^{\left(\beta_{j}\right)}-\gamma_{k+1} C^{T}-G^{T}\right) \phi(x) .
$$


The use of generalized Laguerre tau approximation generates $(N-m+1)$ system of linear equations

$$
\begin{array}{r}
\left\langle R_{N}(x), L_{j}^{(\alpha)}(x)\right\rangle \\
=\int_{0}^{\infty} w(x) R_{N}(x) L_{j}^{(\alpha)}(x) d x=0, \\
\quad j=0,1, \ldots, N-m .
\end{array}
$$

Substituting (23) and (38) into (37) generates $m$ set of linear equations

$$
u^{(i)}(0)=C^{T} \mathbf{D}^{(i)} \phi(0)=d_{i}, \quad i=0,1, \ldots, m-1 .
$$

The combination of (42) and (43) reduces the solution of (36)-(37) to a linear system of algebraic equations, which can be solved for unknown coefficients of the vector $C$ by any direct solver technique to find the spectral solution $u(x)$.

4.2. Nonlinear Multiorder FDEs. In this section, we present the generalized Laguerre pseudo-spectral approximation in combination with GLOM of fractional derivative to find the approximate solution $u_{N}(x)$.

Let us consider the nonlinear multi-term FDE

$$
D^{v} u(x)=F\left(x, u(x), D^{\beta_{1}} u(x), \ldots, D^{\beta_{k}} u(x)\right), \quad \text { in } \Lambda,
$$

subject to the nonhomogeneous initial conditions (37), where $F$ can be nonlinear in general. In [30], the authors studied the existence of solutions of a class of nonlinear FDEs.

Now, we will implement the generalized Laguerre operational matrix for treating this nonlinear problem. To do this, firstly, we approximate $u(x), D^{v} u(x)$, and $D^{\beta_{j}} u(x)$, for $j=1, \ldots, k$ by using (38), (40), respectively, and then the operational matrices formulation of (44) can be expressed as

$$
\begin{aligned}
& C^{T} \mathbf{D}^{(\nu)} \phi(x) \\
& \quad \simeq F\left(x, C^{T} \phi(x), C^{T} \mathbf{D}^{\left(\beta_{1}\right)} \phi(x), \ldots, C^{T} \mathbf{D}^{\left(\beta_{k}\right)} \phi(x)\right) .
\end{aligned}
$$

Also, making use of (38) and (23) in (44) yields

$$
u^{(i)}(0)=C^{T} \mathbf{D}^{(i)} \phi(0)=d_{i}, \quad i=0,1, \ldots, m-1 .
$$

Collocating the operational matrix equation (45) at $(N-m+1)$ nodes of the generalized Laguerre-Gauss quadrature on $\Lambda$,

$$
\begin{gathered}
C^{T} \mathbf{D}^{(\nu)} \phi\left(x_{j}^{(\alpha)}\right) \\
\simeq F\left(x, C^{T} \phi\left(x_{j}^{(\alpha)}\right), C^{T} \mathbf{D}^{\left(\beta_{1}\right)} \phi\left(x_{j}^{(\alpha)}\right),\right. \\
\left.\ldots, C^{T} \mathbf{D}^{\left(\beta_{k}\right)} \phi\left(x_{j}^{(\alpha)}\right)\right) .
\end{gathered}
$$

Combining $(N-m+1)$ algebraic equations (47) with $m$ initial conditions $(46)$ generates a system of $(N+1)$ nonlinear algebraic equations. This system may be evaluated by implementing Newton's iterative method to find the spectral solution $u(x)$.

\section{Numerical Results}

This section considers several numerical examples to demonstrate the accuracy and applicability of the proposed spectral algorithms based on operational matrix of fractional derivatives of generalized Laguerre polynomials. A comparison of the results obtained by adopting different choices of the generalized Laguerre parameter $\alpha$ reveals that the present algorithms are very convenient for all choices of $\alpha$ and produces accurate solutions to multi-term FDEs on semiinfinite interval.

Example 1. Consider the linear FDE

$$
\begin{aligned}
& D^{2} u(x)+D^{3 / 2} u(x)+u(x)=1+x, \\
& u(0)=1, \quad u^{\prime}(0)=1, \quad x \in \Lambda,
\end{aligned}
$$

where $u(x)=1+x$ is the exact solution.

If we apply the operational matrix formulation, the generalized Laguerre spectral tau method with $N=2$, we get

$$
u(x)=c_{0} L_{0}^{(\alpha)}(x)+c_{1} L_{1}^{(\alpha)}(x)+c_{2} L_{2}^{(\alpha)}(x)=C^{T} \phi(x) .
$$

Here, we have

$$
\begin{gathered}
\mathbf{D}^{(1)}=-\left(\begin{array}{lll}
0 & 0 & 0 \\
1 & 0 & 0 \\
1 & 1 & 0
\end{array}\right), \\
\mathbf{D}^{(2)}=\left(\begin{array}{lll}
0 & 0 & 0 \\
0 & 0 & 0 \\
1 & 0 & 0
\end{array}\right), \\
\mathbf{D}^{(3 / 2)}=\left(\begin{array}{ccc}
0 & 0 \\
0 & 0 & 0 \\
S_{3 / 2}(2,0) & S_{3 / 2}(2,1) & S_{3 / 2}(2,2)
\end{array}\right), \\
G=\left(\begin{array}{l}
g_{0} \\
g_{1} \\
g_{2}
\end{array}\right),
\end{gathered}
$$

where $g_{j}$ and $S_{\nu}(i, j)$ are defined in (18) and (27).

Applying variational formulation of the tau method of (48) yields

$$
c_{0}+c_{2}+S_{3 / 2}(2,0)=g_{0} .
$$

The treatment of initial conditions using (43) gives

$$
\begin{gathered}
c_{0}+(\alpha+1) c_{1}+\frac{(\alpha+1)(\alpha+2)}{2} c_{2}=1, \\
-c_{1}-(\alpha+2) c_{2}-1=0
\end{gathered}
$$

Solving the resulted system of algebraic equations (51)(52) provides the unknown coefficients in terms of the parameter $\alpha$ :

$$
c_{0}=\alpha+2, \quad c_{1}=-1, \quad c_{2}=0 .
$$


TABLE 1: $c_{0}, c_{1}$, and $c_{2}$ for different values of $\alpha$ for Example 1 .

\begin{tabular}{lccc}
\hline$\alpha$ & $c_{0}$ & $c_{1}$ & $c_{2}$ \\
\hline-0.5 & 1.5 & -1 & 0 \\
0 & 2 & -1 & 0 \\
0.5 & 2.5 & -1 & 0 \\
1 & 3 & -1 & 0 \\
2 & 4 & -1 & 0 \\
3 & 5 & -1 & 0 \\
\hline
\end{tabular}

Accordingly, the approximate solution can be written as

$$
u(x)=\left(c_{0}, c_{1}, c_{2}\right)\left(\begin{array}{c}
L_{0}^{(\alpha)}(x) \\
L_{1}^{(\alpha)}(x) \\
L_{2}^{(\alpha)}(x)
\end{array}\right)=1+x
$$

which is the exact solution.

Table 1 lists the values of $c_{0}, c_{1}$, and $c_{2}$ with different choices of $(\alpha)$. Indeed, we can achieve the exact solution of this problem with all choices of the generalized Laguerre parameters $\alpha$.

\section{Example 2. Consider linear FED}

$$
\begin{gathered}
D^{2} u(x)+D^{1 / 2} u(x)+u(x) \\
=x^{2}+2+\frac{2.6666666667}{\Gamma(0.5)} x^{1.5}, \\
u(0)=0, \quad u^{\prime}(0)=0, \quad x \in \Lambda .
\end{gathered}
$$

The analytical solution is $u(x)=x^{2}$.

The use of technique described in Section 4.1 with $N=2$ enables one to approximate the solution as

$$
u(x)=c_{0} L_{0}^{(\alpha)}(x)+c_{1} L_{0}^{(\alpha)}(x)+c_{2} L_{2}^{(\alpha)}(x)=C^{T} \phi(x) .
$$

Here, we have

$$
\begin{aligned}
& \mathbf{D}^{(2)}=\left(\begin{array}{lll}
0 & 0 & 0 \\
0 & 0 & 0 \\
1 & 0 & 0
\end{array}\right), \\
& \mathbf{D}^{(1 / 2)}=\left(\begin{array}{ccc}
0 & 0 & 0 \\
S_{1 / 2}(1,0) & S_{1 / 2}(1,1) & S_{1 / 2}(1,2) \\
S_{1 / 2}(2,0) & S_{1 / 2}(2,0) & S_{1 / 2}(2,0)
\end{array}\right), \\
& G=\left(\begin{array}{l}
g_{0} \\
g_{1} \\
g_{2}
\end{array}\right) .
\end{aligned}
$$

Therefore using (42), we obtain

$$
c_{0}+S_{1 / 2}(1,0) c_{1}+\left[S_{1 / 2}(2,0)+1\right] c_{2}=6
$$

TABLE 2: $c_{0}, c_{1}$, and $c_{2}$ for different values of $\alpha$ for Example 2 .

\begin{tabular}{lccc}
\hline$\alpha$ & $c_{0}$ & $c_{1}$ & $c_{2}$ \\
\hline-0.5 & 0.75 & -3 & 2 \\
0 & 2 & -4 & 2 \\
0.5 & 3.75 & -5 & 2 \\
1 & 6 & -6 & 2 \\
2 & 12 & -8 & 2 \\
3 & 20 & -10 & 2 \\
\hline
\end{tabular}

Now, making use of (43) yields

$$
\begin{gathered}
c_{0}+(\alpha+1) c_{1}+\frac{(\alpha+1)(\alpha+2)}{2} c_{2}=0 \\
-c_{1}-(\alpha+2) c_{2}=0
\end{gathered}
$$

Finally by solving (58)-(59), then, we get

$$
c_{0}=\alpha^{2}+3 \alpha+2, \quad c_{1}=-2 \alpha-4, \quad c_{2}=2
$$

Thus we can write

$$
u(x)=\left(c_{0}, c_{1}, c_{2}\right)\left(\begin{array}{c}
L_{0}^{(\alpha)}(x) \\
L_{1}^{(\alpha)}(x) \\
L_{2}^{(\alpha)}(x)
\end{array}\right)=x^{2}
$$

which is the exact solution.

In Table 2, we exhibit the values of $c_{0}, c_{1}$ and $c_{2}$ with different choices of $(\alpha)$.

Example 3. Consider linear initial value problem of fractional order (see [31])

$$
\begin{gathered}
D^{2} u(x)-2 D u(x)+D^{1 / 2} u(x)+u(x) \\
=x^{3}-6 x^{2}+6 x+\frac{16}{5 \sqrt{\pi}} x^{2.5}, \\
u(0)=0, \quad u^{\prime}(0)=0, \quad x \in \Lambda,
\end{gathered}
$$

whose exact solution is given by $u(x)=x^{3}$.

If we apply the operational matrix formulation, the generalized Laguerre spectral tau method with $N=2$, we get

$$
u(x)=\sum_{i=0}^{3} c_{i} L_{i}^{(\alpha)}(x)=C^{T} \phi(x)
$$


Also,

$$
\begin{gathered}
\mathbf{D}^{(2)}=\left(\begin{array}{llll}
0 & 0 & 0 & 0 \\
0 & 0 & 0 & 0 \\
1 & 0 & 0 & 0 \\
2 & 1 & 0 & 0
\end{array}\right), \quad \mathbf{D}^{(1)}=-\left(\begin{array}{llll}
0 & 0 & 0 & 0 \\
1 & 0 & 0 & 0 \\
1 & 1 & 0 & 0 \\
1 & 1 & 1 & 0
\end{array}\right), \\
\mathbf{D}^{(1 / 2)}=\left(\begin{array}{cccc}
0 & 0 & 0 & 0 \\
S_{1 / 2}(1,0) & S_{1 / 2}(1,1) & S_{1 / 2}(1,2) & S_{1 / 2}(1,3) \\
S_{1 / 2}(2,0) & S_{1 / 2}(2,1) & S_{1 / 2}(2,2) & S_{1 / 2}(2,3) \\
S_{1 / 2}(3,0) & S_{1 / 2}(3,1) & S_{1 / 2}(3,2) & S_{1 / 2}(3,3)
\end{array}\right), \\
G=\left(\begin{array}{l}
g_{0} \\
g_{1} \\
g_{2} \\
g_{3}
\end{array}\right) .
\end{gathered}
$$

Therefore using (42), we obtain

$$
\begin{aligned}
& c_{0}+ {\left[2 c_{1}+S_{1 / 2}(1,0)\right] c_{1} } \\
&+ {\left[3+S_{1 / 2}(2,0)\right] c_{2} } \\
&+ {\left[4+S_{1 / 2}(3,0)\right] c_{3}-g_{0}=0, } \\
& {\left[1+S_{1 / 2}(1,1)\right] c_{1} } \\
&+\left[2+S_{1 / 2}(2,1)\right] c_{2} \\
&+\left[3+S_{1 / 2}(3,1)\right] c_{3}-g_{1}=0, \\
& C^{T} \phi(0)= c_{0}+(\alpha+1) c_{1}+\frac{(\alpha+1)(\alpha+2)}{2} c_{2} \\
&+\frac{(\alpha+1)(\alpha+2)(\alpha+3)}{6} c_{3}=0, \\
& C^{T} \mathbf{D}^{(1)} \phi(0)=-c_{1}-(\alpha+2) c_{2}-\frac{(\alpha+3)(\alpha+2)}{2} c_{3}=0 .
\end{aligned}
$$

Accordingly, we get

$$
\begin{aligned}
& c_{0}=\alpha^{3}+6 \alpha+11 \alpha+6, \\
& c_{1}=-3 \alpha^{2}-15 \alpha-18, \\
& c_{2}=6 \alpha+18, \quad c_{3}=-6 .
\end{aligned}
$$

Thus we can write

$$
u(x)=\left(\begin{array}{llll}
c_{0} & c_{1}, & c_{2} & c_{3}
\end{array}\right)\left(\begin{array}{c}
L_{0}^{(\alpha)}(x) \\
L_{1}^{(\alpha)}(x) \\
L_{2}^{(\alpha)}(x) \\
L_{3}^{(\alpha)}(x)
\end{array}\right)=x^{3},
$$

which is the exact solution. The 4 unknown coefficients with various choices of $\alpha$ are listed in Table 3 .
TABLE 3: $c_{0}, c_{1}, c_{2}$, and $c_{3}$ for different values of $\alpha$ for Example 3.

\begin{tabular}{lcccc}
\hline$\alpha$ & $c_{0}$ & $c_{1}$ & $c_{2}$ & $c_{3}$ \\
\hline-0.5 & $15 / 8$ & $-45 / 4$ & 15 & -6 \\
0 & 6 & -18 & 18 & -6 \\
0.5 & $105 / 8$ & $-105 / 4$ & 21 & -6 \\
1 & 24 & -36 & 24 & -6 \\
2 & 60 & -60 & 30 & -6 \\
3 & 120 & -90 & 36 & -6 \\
\hline
\end{tabular}

Example 4. Consider the following FDE:

$$
\begin{gathered}
D^{5 / 2} u(x)+D^{2} u(x)-2 D^{1 / 2} u(x)+4 u(x)=g(x), \\
u(0)=0, \quad u^{\prime}(0)=0, \quad x \in \Lambda,
\end{gathered}
$$

where

$$
g(x)=4 x^{9}+72 x^{7}-\frac{2 \Gamma(10)}{\Gamma(19 / 2)} x^{17 / 2}+\frac{\Gamma(10)}{\Gamma(15 / 2)} x^{13 / 2}
$$

and the exact solution is $u(x)=x^{9}$.

Now, if we use the spectral tau approximation based on with $N=9$ and $x \in \Lambda$, then we obtain

$$
u(x)=\sum_{i=0}^{9} c_{i} L_{i}^{(\alpha)}(x)=x^{9},
$$

which is the exact solution.

Example 5. Consider the nonhomogeneous fractional initial value problem

$$
\begin{aligned}
& D^{2} u(x)+D^{v} u(x)+u(x)=g(x), \\
& u(0)=1, \quad u^{\prime}(0)=0, \quad x \in(0,10),
\end{aligned}
$$

where

$$
\begin{array}{r}
g(x) \\
=v^{6}\left(e^{\left(\left(1 / \nu^{3}\right) x\right)}+\sin \left(\frac{1}{\nu^{3}} x\right)\right)+e^{\left(\left(1 / \nu^{3}\right) x\right)}-\sin \left(\frac{1}{\nu^{3}} x\right) \\
\quad+\frac{1}{\Gamma(-\nu)} \int_{0}^{x}(x-t)^{-\nu-1}\left(e^{\left(\left(1 / \nu^{3}\right) x\right)}-\sin \left(\frac{1}{\nu^{3}} x\right)\right) d t
\end{array}
$$

and the exact solution is given by $u(x)=e^{\left(\left(1 / v^{3}\right) x\right)}-$ $\sin \left(\left(1 / \nu^{3}\right) x\right)$.

Table 4 introduces the maximum absolute errors, using the tau method based on GLOM of fractional derivative, at $\nu=1.5$ and with different choices of the parameters $\alpha$ and $N$. The curves of exact solutions and approximate solutions obtained by the proposed method for $\alpha=1, N=10$, and $\nu=$ 1.5, 1.7, 1.9 are shown in Figures 1 and 2. From these figures, the exact and approximate solutions are completely conciet. 
TABLE 4: Maximum absolute error for various choices of $\alpha$ and $N$ for Example 5 .

\begin{tabular}{|c|c|c|c|c|c|c|c|}
\hline$N$ & $v$ & $\alpha$ & GLOM & $\alpha$ & GLOM & $\alpha$ & GLOM \\
\hline 5 & 1.5 & 0 & $1.69 \cdot 10^{-2}$ & 1 & $4.41 \cdot 10^{-2}$ & 2 & $1.07 \cdot 10^{-1}$ \\
\hline 10 & & & $1.08 \cdot 10^{-3}$ & & $4.17 \cdot 10^{-4}$ & & $6.52 \cdot 10^{-4}$ \\
\hline 15 & & & $4.99 \cdot 10^{-6}$ & & $1.46 \cdot 10^{-5}$ & & $1.33 \cdot 10^{-5}$ \\
\hline 20 & & & $3.57 \cdot 10^{-8}$ & & $1.22 \cdot 10^{-7}$ & & $2.41 \cdot 10^{-7}$ \\
\hline 25 & & & $3.50 \cdot 10^{-10}$ & & $1.30 \cdot 10^{-9}$ & & $4.07 \cdot 10^{-9}$ \\
\hline 30 & & & $4.03 \cdot 10^{-12}$ & & $1.55 \cdot 10^{-11}$ & & $3.47 \cdot 10^{-11}$ \\
\hline 35 & & & $5.10 \cdot 10^{-14}$ & & $2.08 \cdot 10^{-13}$ & & $6.50 \cdot 10^{-13}$ \\
\hline 40 & & & $6.87 \cdot 10^{-16}$ & & $2.64 \cdot 10^{-15}$ & & $5.29 \cdot 10^{-15}$ \\
\hline
\end{tabular}

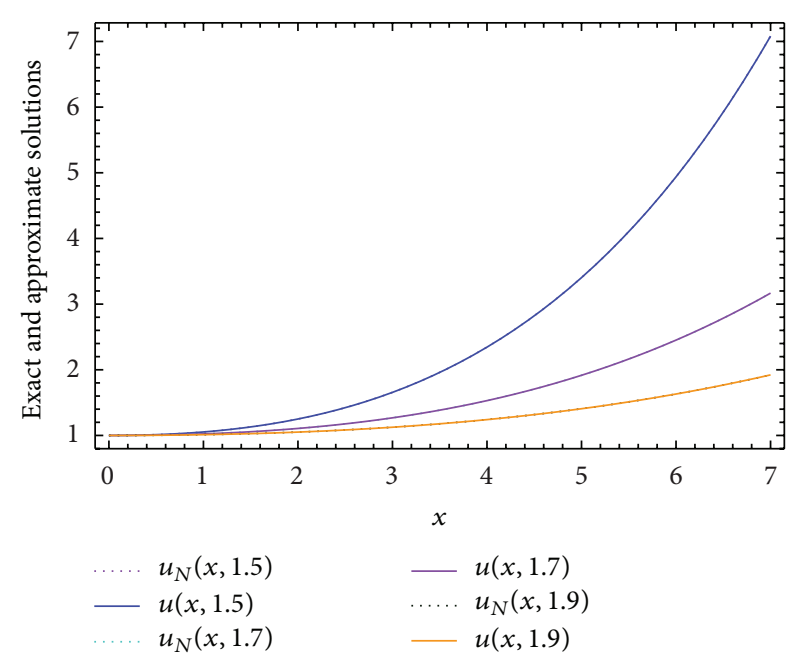

FIgURE 1: Comparing the exact solution and the approximate solutions at $N=10, \alpha=1$, and $v=1.5,1.7,1.9$.

Example 6. Let us consider the nonlinear fractional initial value problem

$$
\begin{aligned}
& D^{2} u(x)+D^{1 / 2} u(x)+u^{2}(x)=g(x), \\
& u(0)=1, \quad u^{\prime}(0)=0, \quad x \in(0,20),
\end{aligned}
$$

where

$$
\begin{aligned}
g(x)= & \cos ^{2}(\gamma x)-\gamma^{2} \cos (\gamma x) \\
& +\frac{1}{\Gamma(-1 / 2)} \int_{0}^{x}(x-t)^{-\nu-1} \cos (\gamma t) d t
\end{aligned}
$$

and the exact solution is given by $u(x)=\cos (\gamma x)$.

The solution of this problem is obtained by applying the generalized Laguerre-Gauss collocation method based on generalized Laguerre operational matrix. The absolute error between the exact and the approximate solution obtained by the proposed method $\gamma=0.01, \alpha=1$ and $N=30$ is given in Figure 3.

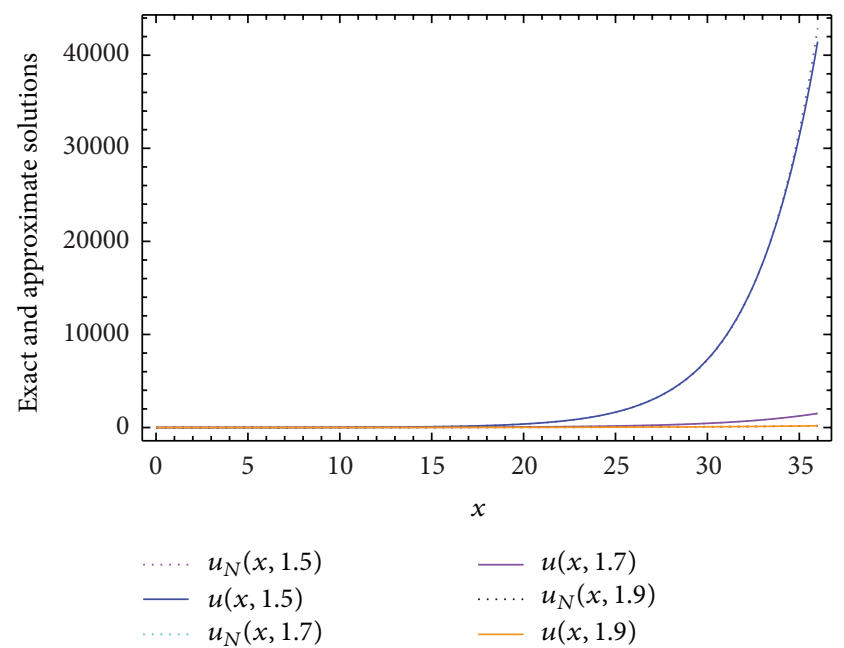

FIGURE 2: Comparing the exact solution and the approximate solutions at $N=10, \alpha=1$, and $\nu=1.5,1.7,1.9$.

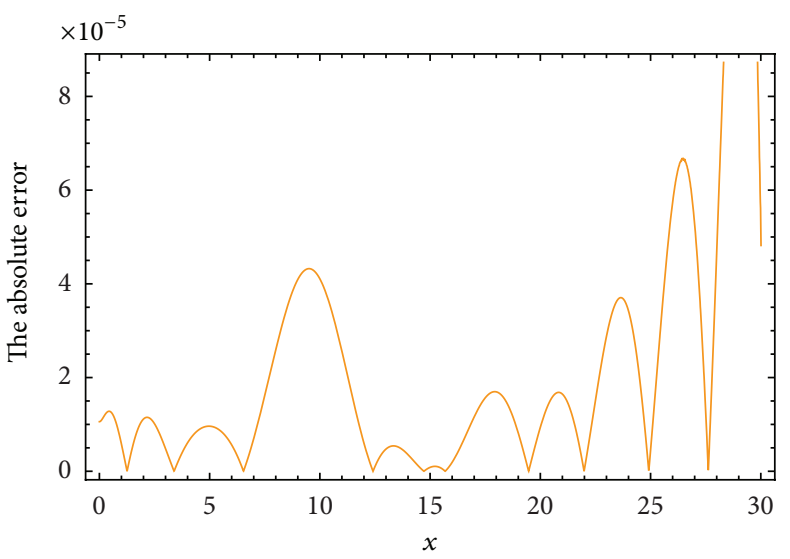

FIGURE 3: The absolute error for $\gamma=1 / 100, \alpha=1$ at $N=30$.

Example 7. We consider the nonlinear fractional initial value problem

$$
\begin{aligned}
& D^{\xi} u(x)+D^{\eta} u(x) D^{\theta} u(x)+u^{2}(x)=g(x), \\
& u(0)=0, \quad u^{\prime}(0)=0, \quad u^{\prime \prime}(0)=0, \quad x \in(0,1), \\
& \zeta \in(2,3), \quad \eta \in(1,2), \quad \theta \in(0,1),
\end{aligned}
$$

where

$$
g(x)=x^{6}+\frac{6 x^{3-\xi}}{\Gamma(4-\xi)}+\frac{36 x^{6-\eta-\theta}}{\Gamma(4-\eta) \Gamma(4-\theta)}
$$

and the exact solution is $u(x)=x^{3}$.

The solution of this problem is obtained by applying the generalized Laguerre-Gauss collocation method based on generalized Laguerre operational matrix for $\xi=2.001, \eta=$ 1.001 , and $\theta=0.001$. The exact solution and approximate 


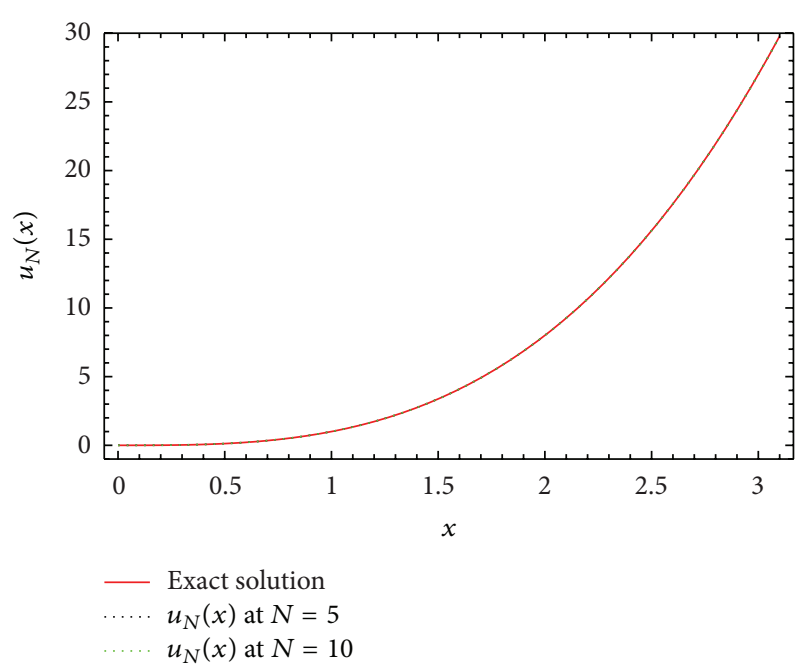

FIgURE 4: Comparing the exact solution and the approximate solutions at $N=5,10$ and $\alpha=1$.

solutions obtained by the proposed method for $\alpha=1$ and two choices of $N$ are shown in Figure 4.

\section{Conclusion}

In this paper, the generalized Laguerre operational matrix of Caputo fractional derivative was derived. Furthermore, we have implemented the generalized Laguerre tau approximation in combination with the GLOM with the generalized Laguerre family to solve the linear FDEs. In addition, combining the pseudo-spectral approximation and the GLOM of fractional derivative was applied to develop an accurate approximate solution of nonlinear FDEs. The generalized Laguerre-Gauss quadrature points were used as a collocation nodes. This method reduces the nonlinear FDEs to a system of algebraic equation in the expansion coefficients which can be solved by any standard technique. The numerical results demonstrate that the proposed spectral algorithms are accurate and efficient.

\section{References}

[1] D. Baleanu, K. Diethelm, E. Scalas, and J. J. Trujillo, Fractional Calculus: Models and Numerical Methods, vol. 3 of Series on Complexity, Nonlinearity and Chaos, World Scientific Publishing, Hackensack, NJ, USA, 2012.

[2] K. S. Miller and B. Ross, An Introduction to the Fractional Calculus and Fractional Differential Equations, John Wiley \& Sons, New York, NY, USA, 1993.

[3] M. D. Ortigueira, "Introduction to fractional linear systems. Part 1: continuous-time case," IEE Proceedings: Vision, Image and Signal Processing, vol. 147, no. 1, pp. 62-70, 2000.

[4] S. Das, Functional Fractional Calculus for System Identification and Controls, Springer, New York, NY, USA, 2008.

[5] C. Canuto, M. Y. Hussaini, A. Quarteroni, and T. A. Zang, Spectral Methods in Fluid Dynamics, Springer, New York, NY, USA, 1988.
[6] E. H. Doha, A. H. Bhrawy, and S. S. Ezz-Eldien, "A Chebyshev spectral method based on operational matrix for initial and boundary value problems of fractional order," Computers \& Mathematics with Applications, vol. 62, no. 5, pp. 2364-2373, 2011.

[7] E. H. Doha, A. H. Bhrawy, and S. S. Ezz-Eldien, "A new Jacobi operational matrix: an application for solving fractional differential equations," Applied Mathematical Modelling, vol. 36, no. 10, pp. 4931-4943, 2012.

[8] D. Funaro, "Estimates of Laguerre spectral projectors in Sobolev spaces," in Orthogonal Polynomials and Their Applications, C. Brezinski, L. Gori, and A. Ronveaux, Eds., vol. 9, pp. 263-266, Scientific Publishing, Singapore, 1991.

[9] A. H. Bhrawy, A. S. Alofi, and S. S. Ezz-Eldien, "A quadrature tau method for fractional differential equations with variable coefficients," Applied Mathematics Letters, vol. 24, no. 12, pp. 2146-2152, 2011.

[10] J. Song, F. Yin, X. Cao, and F. Lu, "Fractional variational iteration method versus Adomian's decomposition method in some fractional partial differential equations," Journal of Applied Mathematics, vol. 2013, Article ID 392567, 10 pages, 2013.

[11] H. Jafari, H. Tajadodi, and D. Baleanu, "A modified variational iteration method for solving fractional Riccati differential equation by Adomian polynomials," Fractional Calculus and Applied Analysis, vol. 16, no. 1, pp. 109-122, 2013.

[12] A. A. Hemeda, "New iterative method: an application for solving fractional physical differential equations," Abstract and Applied Analysis, vol. 2013, Article ID 617010, 9 pages, 2013.

[13] G.-C. Wu and D. Baleanu, "Variational iteration method for fractional calculus: a universal approach by Laplace transform," Advances in Difference Equations, vol. 2013, article 18, 2013.

[14] C. Yang and J. Hou, "An approximate solution of nonlinear fractional differential equation by Laplace transform and adomian polynomials," Journal of Information and Computational Science, vol. 10, no. 1, pp. 213-222, 2013.

[15] M. Gulsua, Y. Ozturka, and A. Anapalia, "Numerical approach for solving fractional Fredholm integro-differential equation," International Journal of Computer Mathematics, 2013.

[16] H. S. Nik, S. Effati, S. S. Motsa, and S. Shateyi, "A new piecewisespectral homotopy analysis method for solving chaotic systems of initial value problems," Mathematical Problems in Engineering, vol. 2013, Article ID 583193, 13 pages, 2013.

[17] E. H. Doha, A. H. Bhrawy, D. Baleanu, and S. S. Ezz-Eldien, "On shifted Jacobi spectral approximations for solving fractional differential equations," Applied Mathematics and Computation, vol. 219, no. 15, pp. 8042-8056, 2013.

[18] M. Maleki, I. Hashim, M. Tavassoli Kajani, and S. Abbasbandy, "An adaptive pseudospectral method for fractional order boundary value problems," Abstract and Applied Analysis, vol. 2012, Article ID 381708, 19 pages, 2012.

[19] A. Al-Rabtah, S. Momani, and M. A. Ramadan, "Solving linear and nonlinear fractional differential equations using spline functions," Abstract and Applied Analysis, vol. 2012, Article ID 426514, 9 pages, 2012.

[20] A. H. Bhrawy, M. M. Alghamdi, and T. M. Taha, "A new modified generalized Laguerre operational matrix of fractional integration for solving fractional differential equations on the half line," Advances in Difference Equations, vol. 2012, article 179, 2012.

[21] S. Yuzbasi, "Numerical solution of the Bagley-Torvik equation by the Bessel collocation method," Mathematical Methods in the Applied Sciences, vol. 36, no. 3, pp. 300-312, 2013. 
[22] M. Shaban, S. Kazem, and J. A. Rad, "A modification of the homotopy analysis method based on Chebyshev operational matrices," Mathematical and Computer Modelling, vol. 57, pp. 1227-1239, 2013.

[23] K. Parand and S. A. Kaviani, "Application of the exact operational matrices based on the Bernstein polynomials," Journal of Mathematics and Computer Science, vol. 6, pp. 36-59, 2013.

[24] E. Tohidi, F. Soleymani, and A. Kilicman, "Robustness of operational matrices of differentiation for solving state-space analysis and optimal control problems," Abstract and Applied Analysis, vol. 2013, Article ID 535979, 9 pages, 2013.

[25] A. H. Bhrawy, D. Baleanu, L. M. Assas, and J. A. Tenreiro Machado, "On a generalized laguerre operational matrix of fractional integration," Mathematical Problems in Engineering, vol. 2013, Article ID 569286, 7 pages, 2013.

[26] M. El-Kady and A. El-Sayed, "Fractional differentiation matrices for solving fractional orders differential equations," International Journal of Pure and Applied Mathematics, vol. 84, no. 2, pp. 1-13, 2013.

[27] G. Szego, Orthogonal Polynomials, American Mathematical Society, Providence, RI, USA, 4th edition, 1975.

[28] D. Funaro, Polynomial Approximation of Differential Equations, vol. 8, Springer, Berlin, Germany, 1992.

[29] D. K. Dimitrov, F. Marcellán, and F. R. Rafaeli, "Monotonicity of zeros of Laguerre-Sobolev-type orthogonal polynomials," Journal of Mathematical Analysis and Applications, vol. 368, no. 1, pp. 80-89, 2010.

[30] D. Baleanu, H. Mohammadi, and S. Rezapour, "Positive solutions of an initial value problem for nonlinear fractional differential equations," Abstract and Applied Analysis, vol. 2012, Article ID 837437, 7 pages, 2012.

[31] E. A. Rawashdeh, "Numerical solution of fractional integrodifferential equations by collocation method," Applied Mathematics and Computation, vol. 176, no. 1, pp. 1-6, 2006. 


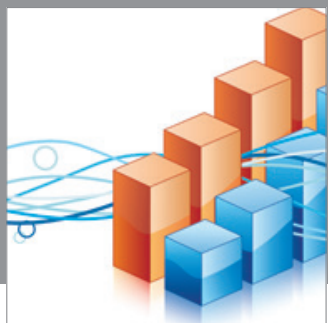

Advances in

Operations Research

mansans

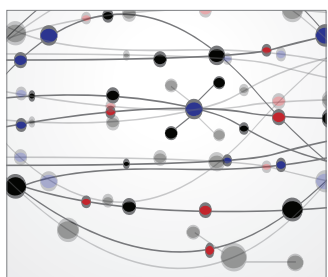

The Scientific World Journal
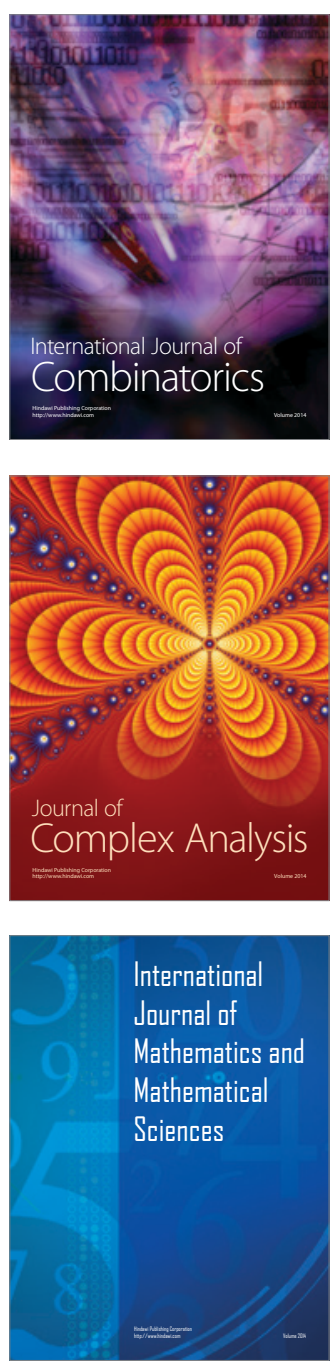
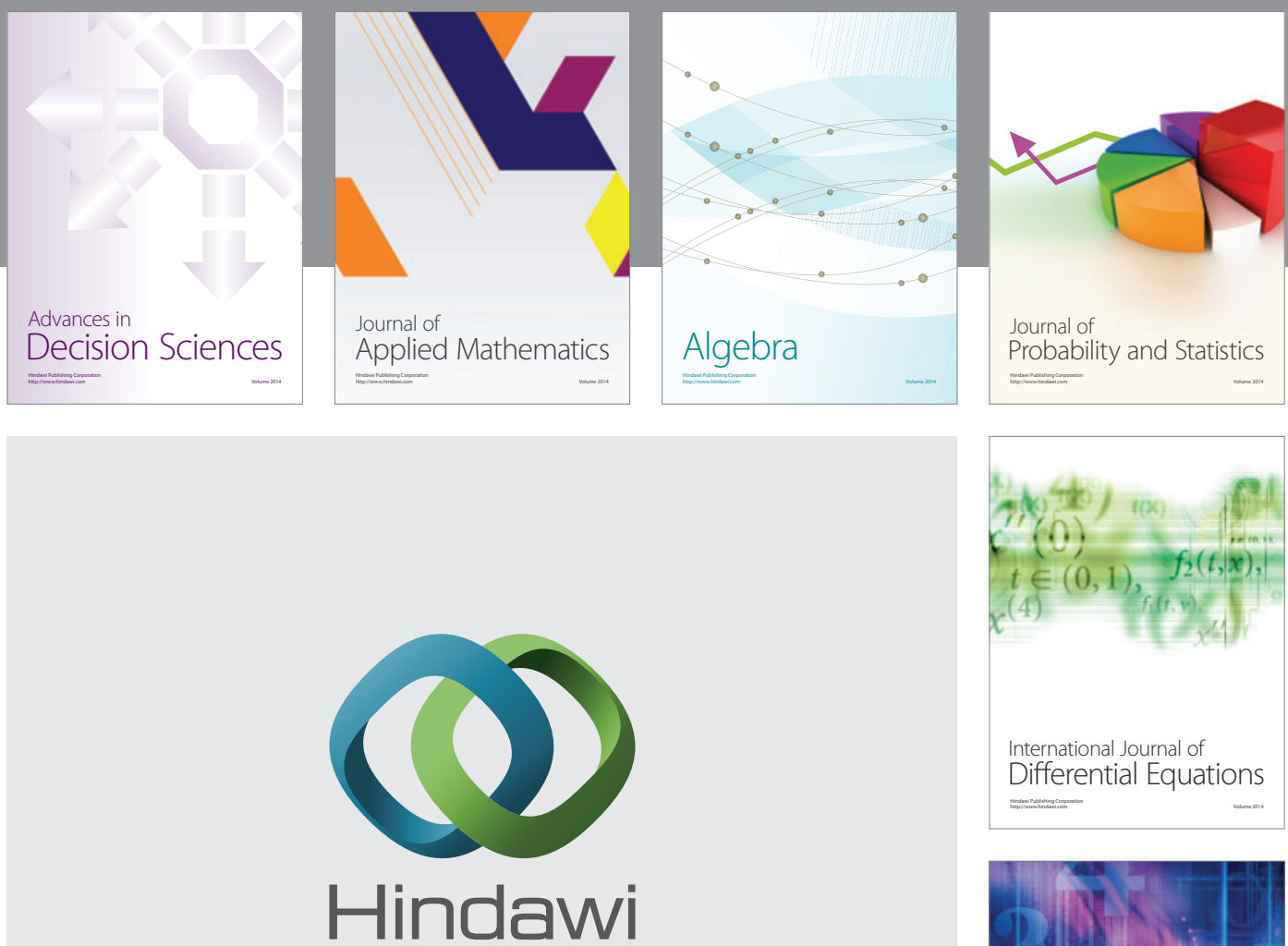

Submit your manuscripts at http://www.hindawi.com
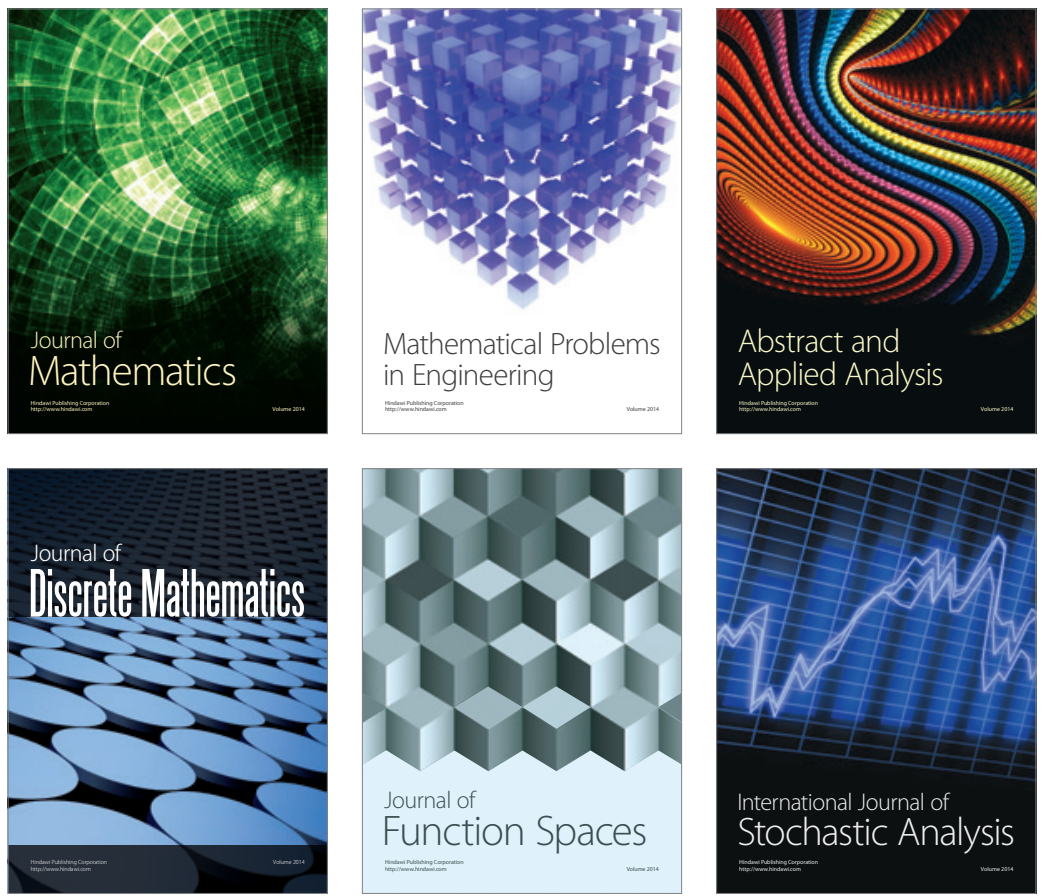

Journal of

Function Spaces

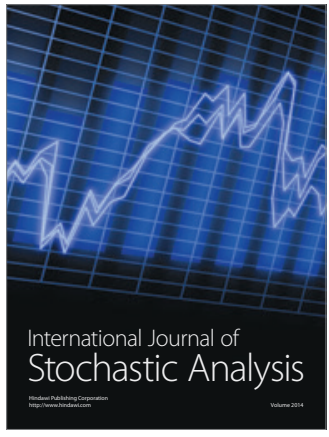

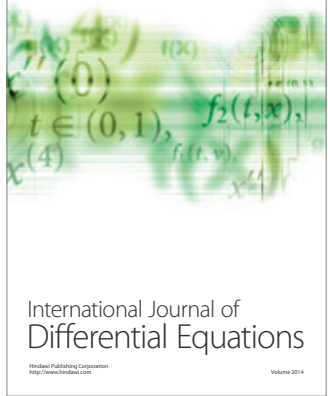
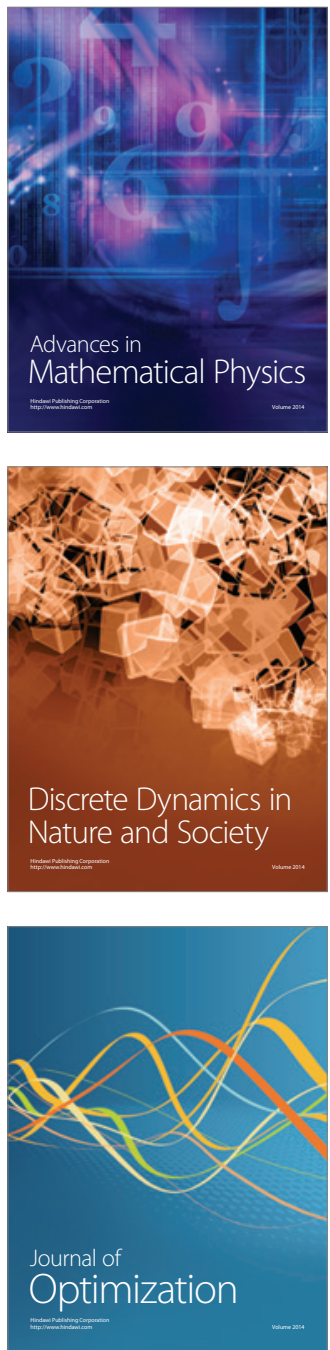